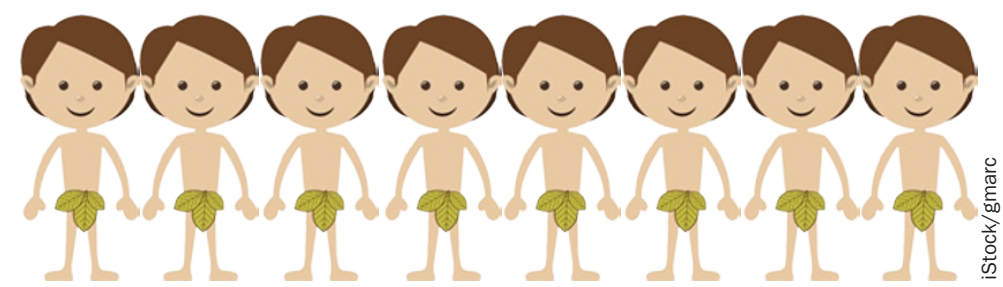

GASTROINTESTINAL CANCER

\title{
ADAM8 provides new hope in pancreatic cancer
}

Pancreatic Ductal Adenocarcinoma (PDAC) is the most common form of pancreatic cancer with high mortality and a 5-year survival rate $<5 \%$. The majority of PDACs (95\%) are associated with mutations in the KRAS gene. Notably, a poor prognosis for patients with this disease has also been correlated with elevated expression levels of ADAM8, a metalloproteinase-disintegrin that is upregulated in PDAC cells, and could provide a new target for the treatment of PDAC as indicated by Jörg-Walter Bartsch and colleagues in their recent publication.

In this study, an international team of researchers investigated the role of ADAM8 in PDAC, reporting that ADAM8 expression is associated with increased migration and invasiveness of PDAC cells owing to the activation of ERK1/2 signalling and to increased extracellular activities of other metalloproteinases.

Synthesized in a proform, ADAM8 needs to multimerize to exert its biological function. The researchers, therefore, designed a peptidomimetic inhibitor, BK-1361, that prevents ADAM8 multimerization and sought to validate this inhibitor. "We employed biochemical methods to characterize the function of the ADAM8 inhibitor and the effect of this inhibition on tumour-cell signalling," explains Bartsch. He continues, "we used different mouse models of PDAC, initially implanting genetically engineered human pancreatic cancer cells in the pancreas of mice and then using a genetic mouse model of PDAC that matched the human pathology."

Application of BK-1361 in these PDAC mouse models resulted in a reduction of tumor burden, infiltration and metastasis by affecting downstream signalling of ADAM8, as indicated by the consequent decrease in ERK1/2 and matrix metalloproteinase activities. Of note, a considerably prolonged survival was observed upon ADAM8 inhibition in the mouse model of PDAC harbouring the KRAS LSL-G12D mutation, genetically resembling the human disease. These results have established ADAM8 as a new drug target in pancreatic cancer. "Since ADAM8 is dispensable for the organism as suggested by ADAM8deficient mice, it is an excellent target for therapeutic intervention," says Bartsch.

The researchers are optimising the inhibitor to enable effective blockade of human ADAM8 function. "We will then try to launch preclinical tests in patients with PDAC to check if ADAM8 inhibition is as effective as in mice," concludes Bartsch.

Alessia Errico

Original article Schlomann, U. et al. ADAM8 as a drug target in pancreatic cancer. Nat. Comm. doi:10.1038/ncomms7175 